\title{
Development of a Rat Model for Evaluating Thyroid-Stimulating Hormone Suppression after Total Thyroidectomy
}

\author{
Shan $\operatorname{Jin}^{1,2}$ and Iwao Sugitani ${ }^{2}$ \\ ${ }^{1}$ Department of General Surgery, Affiliated Hospital of Inner Mongolia Medical University, Inner Mongolia Autonomous Region, China \\ ${ }^{2}$ Department of Endocrine Surgery, Graduate School of Medicine, Nippon Medical School, Tokyo, Japan
}

Background: We developed an animal model for evaluating thyroid-stimulating hormone (TSH) suppression therapy after total thyroidectomy in rats.

Methods: Sixty Wistar rats were randomly divided into 6 groups, including a sham-operated group (SO group), a total thyroidectomy group (TD group), and a L-thyroxine (L-T4) treatment I group (TS-I group), II group (TS-II group), III group (TS-III group), and IV group (TS-IV group) (in which rats were treated with 1.4, 1.6, 1.8, and $2.0 \mu \mathrm{g} / 100 \mathrm{~g}$ body weight, respectively) after total thyroidectomy.

Results: HE staining in the TD group and all L-T4-treated rats showed that the resected tissue was normal thyroid gland. No residual thyroid tissue was found in neck tissue of the cross-section of the thyroid gland. Serum T3 levels in the TS-II group were not significantly different from those in the SO group, whereas serum T4 levels were slightly higher than those in the SO group, and serum TSH levels were slightly lower.

Conclusions: Rats subcutaneously injected with L-T4 $1.6 \mu \mathrm{g} / 100 \mathrm{~g}$ body weight for 15 days after total thyroidectomy were suitable as an animal model for TSH suppression therapy.

(J Nippon Med Sch 2021; 88: 311-318)

Key words: animal model, thyroidectomy, TSH suppression therapy

\section{Introduction}

Thyroid cancer is a common malignant endocrine tumor, and its incidence is increasing annually ${ }^{1}$. Oral administration of L-thyroxine (L-T4) for inhibition of thyroidstimulating hormone (TSH) is a standard procedure for postoperative treatment of differentiated thyroid cancer $(\mathrm{DTC})^{2}$. In postoperative TSH suppression therapy for DTC, thyroid hormone tablets are used to suppress TSH at or below the lower limit of the normal range after surgery. This helps address thyroid hormone deficiency after surgery and inhibits growth of cancer cells ${ }^{3}$.

The TSH inhibitory level is closely associated with recurrence, metastasis, and cancer-related death of DTC, especially in high-risk DTC patients. An increase in serum TSH level might promote postoperative DTC progression ${ }^{4}$. The overall prognosis for low-risk DTC patients is significantly improved when TSH level is suppressed to
0.1 to $0.5 \mathrm{mU} / \mathrm{L}$ after surgery. When TSH level in highrisk DTC patients is suppressed to below $0.1 \mathrm{mU} / \mathrm{L}$, tumor recurrence and metastasis are significantly reduced ${ }^{5}$. However, long-term TSH suppression therapy in DTC patients can lead to drug-induced subclinical hyperthyroidism, which significantly increases mortality risk and the incidence of cardiovascular events in adults, as well as the incidence of osteoporosis and fracture in postmenopausal women ${ }^{6,7}$. Cognitive impairment has been reported in some patients ${ }^{8}$. To clarify the effects of TSH suppression therapy on thyroid cancer after thyroidectomy, it is essential to create animal models that facilitate study of the relevant mechanisms. We evaluated an animal model of TSH suppression therapy after total thyroidectomy in Wistar rats by resecting thyroid tissues and administering L-T4.

Correspondence to Iwao Sugitani, Department of Endocrine Surgery, Graduate School of Medicine, Nippon Medical School, 11-5 Sendagi, Bunkyo-ku, Tokyo 113-8603, Japan

E-mail: isugitani@nms.ac.jp

https://doi.org/10.1272/jnms.JNMS.2021_88-409

Journal Website (https://www.nms.ac.jp/sh/jnms/) 


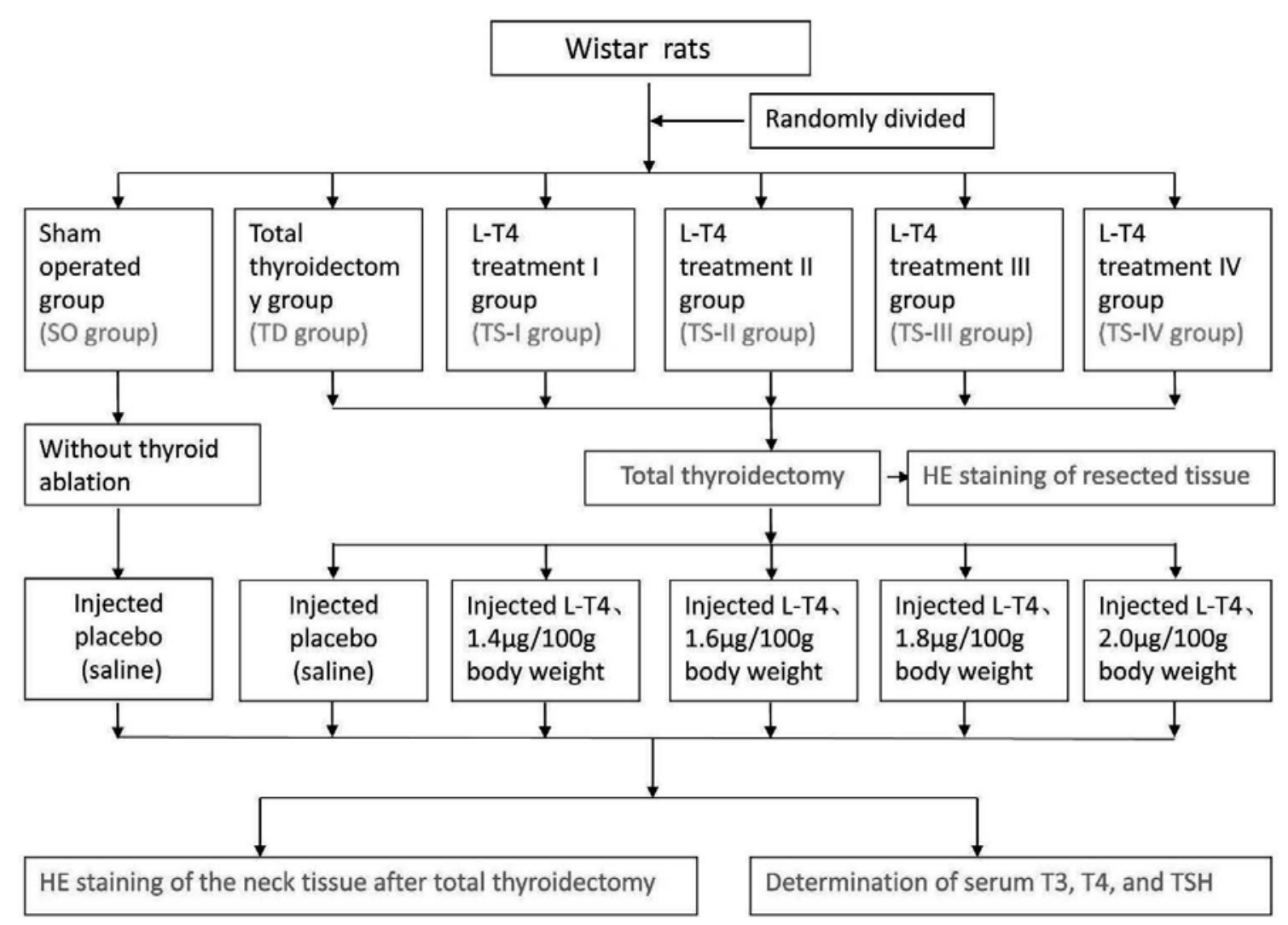

Fig. 1 Overview of research design and experimental process

\section{Materials and Methods}

\section{Ethics Statement}

All research animals were treated in accordance with the Principles of Laboratory Animal Care formulated by the US National Society for Medical Research and the US Guide for the Care and Use of Laboratory Animals published. This study was approved by the biomedical ethics committee of Inner Mongolia Medical University (No. YKD2014063).

\section{Experimental Animal}

Here, clean-grade, female Wistar rats aged 8 weeks with an average body weight (BW) of $200 \pm 30 \mathrm{~g}$ were purchased from the Laboratory Animal Center of Inner Mongolia Medical University (Inner Mongolia, China; Production License No. SCXK [Mongolia] 2015-0001). Feeding conditions were illumination time, 12/12 h, relative humidity, $45 \%$ to $50 \%$, and ambient temperature, $22^{\circ} \mathrm{C}$ to $23^{\circ} \mathrm{C}$. All rats were free to drink and eat.

The rats were randomly assigned to 6 groups. Figure 1 shows the research design and experimental process.

(1) Sham-operated group (SO group): 10 rats were fed a standard diet and distilled water. After the rats were anesthetized with $10 \%$ chloral hydrate solution, the neck was incised to expose the thyroid gland without thyroid ablation, and then suturing was performed.
(2) Total thyroidectomy group (TD group): After 10 rats were adaptively fed for 1 week, total thyroidectomy was performed. The rats were fed a standard diet and highcalcium water ( $1 \%$ calcium gluconate). On the $1^{\text {st }}$ day after surgery, the abdominal subcutaneous injection of placebo was given daily.

(3) L-T4 treatment I group I (TS-I group): After 10 rats were adaptively fed for 1 week, total thyroidectomy was performed. The rats were fed a standard diet and highcalcium water ( $1 \%$ calcium gluconate). On the $1^{\text {st }}$ day after surgery, an abdominal subcutaneous injection of L-T4 $1.4 \mu \mathrm{g} / 100 \mathrm{~g}$ BW was given daily.

(4) L-T4 treatment II group (TS-II group): After 10 rats were adaptively fed for 1 week, total thyroidectomy was performed. The rats were fed a standard diet and highcalcium water ( $1 \%$ calcium gluconate). On the $1^{\text {st }}$ day after surgery, an abdominal subcutaneous injection of L-T4 $1.6 \mu \mathrm{g} / 100 \mathrm{~g}$ BW was given daily.

(5) L-T4 treatment III group (TS-III group): After 10 rats were adaptively fed for 1 week, total thyroidectomy was performed. Rats were fed a standard diet and highcalcium water ( $1 \%$ calcium gluconate). On the $1^{\text {st }}$ day after surgery, an abdominal subcutaneous injection of L-T4 $1.8 \mu \mathrm{g} / 100 \mathrm{~g}$ BW was given daily.

(6) L-T4 treatment IV group (TS-IV group): After 10 
rats were adaptively fed for 1 week, total thyroidectomy was performed. The rats were fed a standard diet and high-calcium water ( $1 \%$ calcium gluconate). On the $1^{\text {st }}$ day after surgery, an abdominal subcutaneous injection of L-T4 $2.0 \mu \mathrm{g} / 100 \mathrm{~g}$ BW was given daily.

Reference Ranges for Thyroid Function in Normal Rats

A previous study established normal reference values for serum thyroid hormones in adult Wistar rats, namely, 0.73 to $0.98 \mathrm{ng} / \mathrm{mL}$ for $\mathrm{T} 3,4.2$ to $8.4 \mathrm{ng} / \mathrm{mL}$ for $\mathrm{T} 4$, and 0.76 to $1.29 \mu \mathrm{IU} / \mathrm{mL}$ for $\mathrm{TSH}^{9}$.

\section{Procedure for Total Thyroidectomy in Rats}

As in our previous study ${ }^{10}$, a longitudinal incision, 2.0 to $2.5 \mathrm{~cm}$ in length, was made in the middle of the neck. Neck skin and subcutaneous connective tissue were incised. The sternohyoid muscle of the trachea was bluntly separated along the middle line. After being opened with a distractor, the white trachea and a pair of oval thyroid glands attached to the thyroid cartilage on both sides of the trachea ring could be seen. The bilateral thyroid glands were deep reddish brown and connected across the trachea by the isthmus (Fig. 2A). The bilateral superior thyroid arteries were ligated, the thyroid isthmus was pinched with tweezers, and the thyroid isthmus was cut with ophthalmic scissors (Fig. 2B). One side of the broken end was lifted, and the thyroid gland was bluntly separated close to the trachea surface; thus, the recurrent laryngeal nerve between the thyroid gland and trachea could be observed. After the recurrent laryngeal nerve was carefully stripped, the unilateral thyroid gland and isthmus were completely resected. The contralateral thyroid gland was then removed with the same technique until there was no thyroid tissue below the thyroid cartilage (Fig. 2C $\sim$ E). Care should be taken to avoid damaging blood vessels intraoperatively, and sterile cotton swab or gauze should be used to immediately stop any intraoperative bleeding.

After the operation, the resected thyroid tissue was fixed in a $10 \%$ neutral solution of formaldehyde (Fig. 2F). After confirming the absence of bleeding, the distractor in the neck was removed, the sternohyoid muscle of the trachea was reset, the muscle membrane and skin were sutured layer-by-layer, the incision was covered with gauze after iodophor disinfection, and warm light was used until recovery.

TSH Suppression Was Induced in the Rat Model after Total Thyroidectomy

Starting on the $1^{\text {st }}$ day after surgery, rats in the SO group and TD group were subcutaneously injected with placebo (saline) daily. Rats in groups I, II, III, and IV were treated with L-T4. From the $1^{\text {st }}$ day after surgery, LT4 was subcutaneously injected into the abdomen daily at doses of 1.4, 1.6, 1.8, and $2.0 \mu \mathrm{g} / 100 \mathrm{~g}$ BW, respectively. After 15 days of injection, the rats were sacrificed and serum samples were collected. Simultaneously, the neck was cut off at the upper and lower $1 \mathrm{~cm}$ of the thyroid location, and neck tissue in the cross-section of thyroid gland was obtained.

\section{Criteria to Determine Model Success}

The rat model for TSH suppression therapy after total thyroidectomy was considered successful when serum T3 and T4 in the L-T4 treatment groups were within the normal ranges specified above, or T4 was slightly elevated and TSH was below the lower limit of the normal range.

\section{Assessment of Indexes and Methods}

Determination of serum T3, T4, and TSH

On the $15^{\text {th }}$ day after surgery, serum samples were collected from rats in the TD group and all L-T4 treated rats, and serum T3, T4, and TSH were then determined with a Cobas E601 automatic electrochemiluminescence immunoassay system (Roche).

Hematoxylin and eosin (HE) staining of resected tissues and neck tissue

Resected neck tissues of rats in the TD group and all L-T4 treated rats were fixed with $10 \%$ formaldehyde neutral solution, dehydrated, and embedded in paraffin. After obtaining a routine $4-\mu \mathrm{m}$ section, HE staining was performed. At the same time, neck tissues of the crosssection of thyroid gland from rats in the SO group and thyroidectomized rats were removed and stained with HE.

\section{Statistical Analysis}

SPSS 19.0 software (IBM, Armonk, NY, USA) was used for statistical analysis. The $\mathrm{W}$-test was used to analyze the normality of each index. Normally distributed data are shown as mean $\pm \mathrm{SD}(X \pm S)$. The t-test was used for comparing those 2 groups, and analysis of variance (ANOVA) was used for comparison among multiple groups. A P value of $<0.05$ was considered to indicate statistical significance.

\section{Results}

\section{HE Staining of Resected Neck Tissue}

HE staining was used to examine resected neck tissue from rats in the TD group and all L-T4 treated rats. Light microscopy revealed numerous round or irregular follicular structures. The follicles were rich in glia, and capillar- 

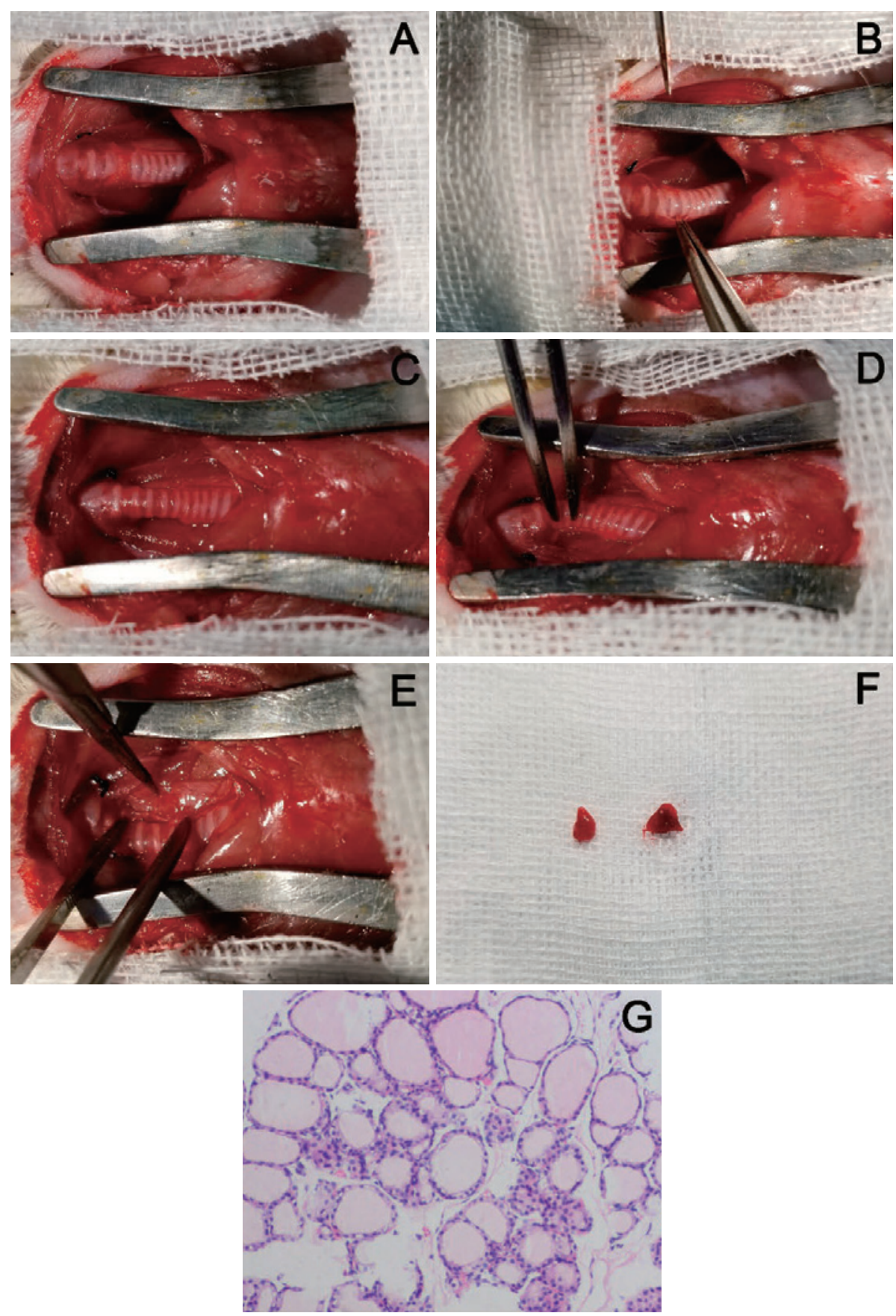

Fig. 2 Images of thyroidectomy and thyroid grand histology in rat A: Anatomical appearance of thyroid gland (the superior thyroid arteries are ligated on both sides). B: Total thyroidectomy. C: Anatomic appearance of neck after total thyroidectomy. D: Exposed right recurrent laryngeal nerve after total thyroidectomy. E: Exposed left recurrent laryngeal nerve after total thyroidectomy. F: Resected thyroid glands. G: Thyroid grand histology $(\mathrm{HE}, 10 \times 10)$.

ies and scattered parafollicular cells were present among the follicles, which proved that the excised tissue was normal rat thyroid gland (Fig. 2G).

HE Staining of a Neck Cross-Section after Total Thyroidectomy

Follicular structure, tracheal cartilage, connective tissue, and muscle tissue were observed in neck tissue of a thyroid gland cross-section of rats in the SO group after HE staining (Fig. 3A). However, no follicular structure was found in the thyroidectomized rats, although tracheal cartilage, connective tissue, muscle, and inflammatory cells infiltrated the resected area, indicating that the thyroid gland was completely resected (Fig. 3B). 


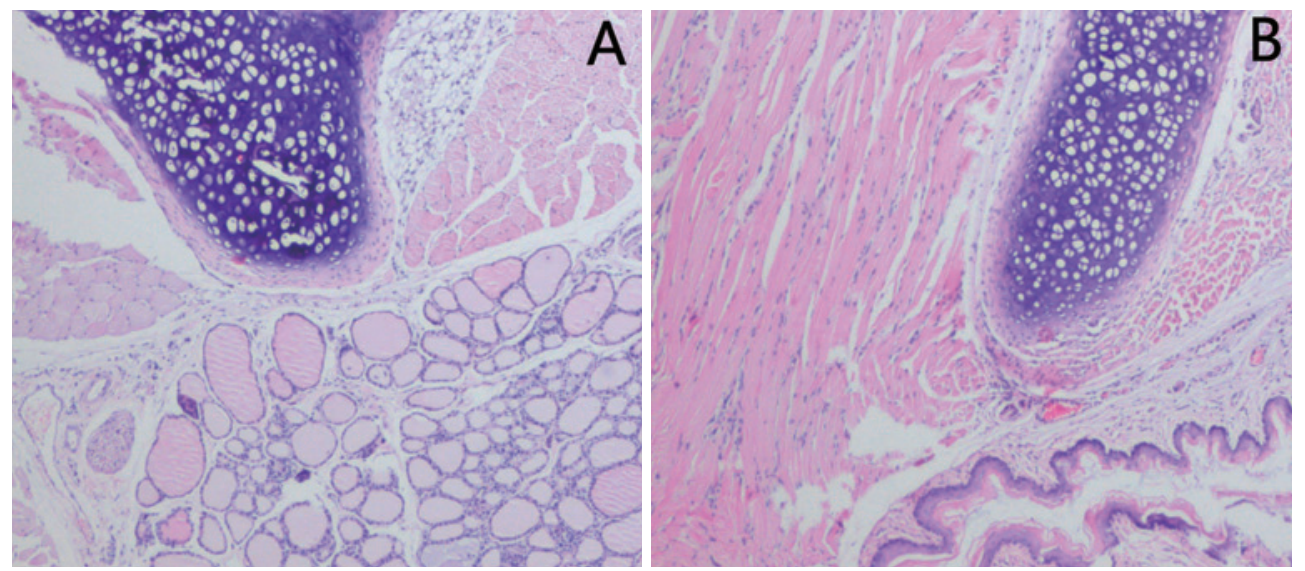

Fig. 3 Histology of the cross-section of neck

A: Histology of cross-section of thyroid gland in a normal rat $(\mathrm{HE}, 4 \times 10)$.

B: Histology of cross-section of thyroid gland after total thyroidectomy $(\mathrm{HE}, 4 \times 10)$.

\section{Serum Thyroid Hormone Levels}

Serum levels of T3 and T4 were significantly lower in the TD group than in the SO group, whereas serum TSH was significantly higher $(\mathrm{P}<0.01)$. Serum levels of T3 and TSH were not significantly different in the TS-I group and SO group ( $\mathrm{P}>0.05)$, while serum $\mathrm{T} 4$ level was slightly higher than that in the SO group $(\mathrm{P}<0.05)$. Serum T3 levels in the TS-II group were not significantly different from those in the SO group $(\mathrm{P}>0.05)$, while serum T4 level was slightly higher than that in the SO group, and serum TSH level was slightly lower than that in the SO group $(\mathrm{P}<0.01)$. Serum levels of $\mathrm{T} 3$ and $\mathrm{T} 4$ were much higher in the TS-III group than in the SO group, whereas the serum level of TSH was significantly lower than that in the SO group $(\mathrm{P}<0.01)$. Serum T3 and T4 levels were significantly higher in the TS-IV group than in the SO group; however, serum TSH level was significantly lower than that in the $\mathrm{SO}$ group $(\mathrm{P}<0.01)$ (Fig. 4).

In sum, after thyroidectomy, rats in the TD group were hypothyroid; rats in the TS-I group were in a replacement state of thyroid function; rats in the TS-II group were in a TSH suppression state; and rats in the TS-III group and TS-IV group were hyperthyroid. Therefore, injection of L-T4 $1.6 \mu \mathrm{g} / 100 \mathrm{~g}$ BW was considered a successful rat model for TSH suppression therapy after total thyroidectomy.

\section{Discussion}

In this study, to evaluate TSH suppression therapy after surgery for DTC, we created a rat model for TSH suppression therapy after total thyroidectomy. To treat DTC patients with TSH suppression therapy after thyroidec- tomy, complete resection of thyroid tissue should first be done, to reduce the effect of residual thyroid tissue on subsequent experiments. The thyroid gland is located in the ventrolateral part of the first 4 or 5 tracheal rings below the thyroid cartilage, and the left and right lateral lobes are connected by the isthmus across the ventral surface of the trachea. The glands are reddish brown, about 3.9 to $5.5 \mathrm{~mm}$ in length and 2.0 to $3.0 \mathrm{~mm}$ in width, and encompass about 4 to 5 tracheal rings. The thyroid and parathyroid glands cannot be distinguished by the naked eye.

The thyroid blood supply was very rich, and there were extensive anastomoses in arteries, from the capsule with connective tissue into the gland. A fenestrated capillary network was formed around the follicles, and then the capillary network reintegrated into the venous network, resulting in greater blood loss during surgical resection $^{11}$. In the rat neck, the bilateral recurrent laryngeal nerves are in the tracheoesophageal groove. The recurrent laryngeal nerve innervates movement of the vocal cords and distributes to most of the larynx, which is responsible for sensation in the laryngeal muscles and mucosa below the vocal cords. Unilateral recurrent laryngeal nerve injury manifests as vocal cord paralysis, while bilateral recurrent laryngeal nerve manifests as aphonia, dyspnea, and even death from asphyxia ${ }^{12}$. In this study, the superior thyroid artery was initially ligated, after which the thyroid isthmus was cut off, and the thyroid gland was fully removed by blunt dissection. This reduced the amount of residual thyroid tissue caused by sharp resection, controlled intraoperative bleeding, and protected the recurrent laryngeal nerve. Excessive force should be avoided during the operation, to avoid crush- 
T3

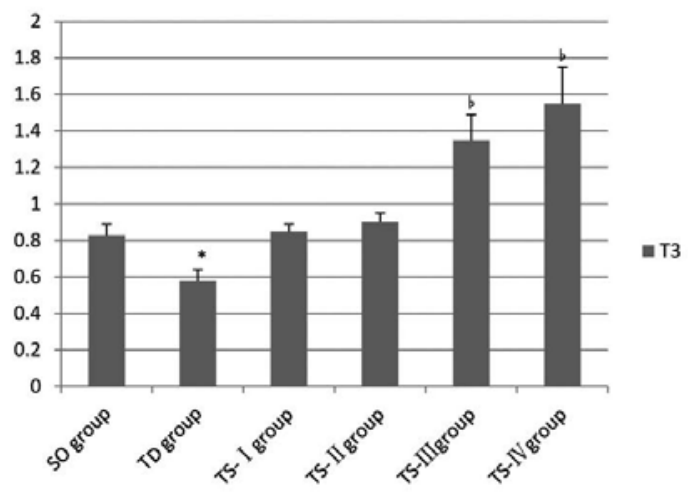

T4

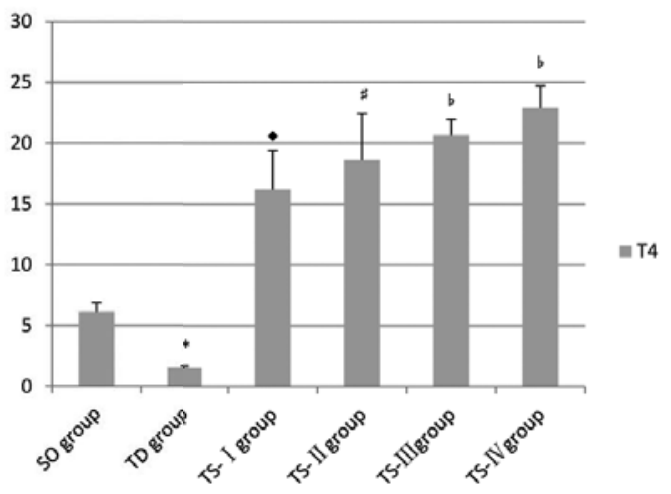

TSH

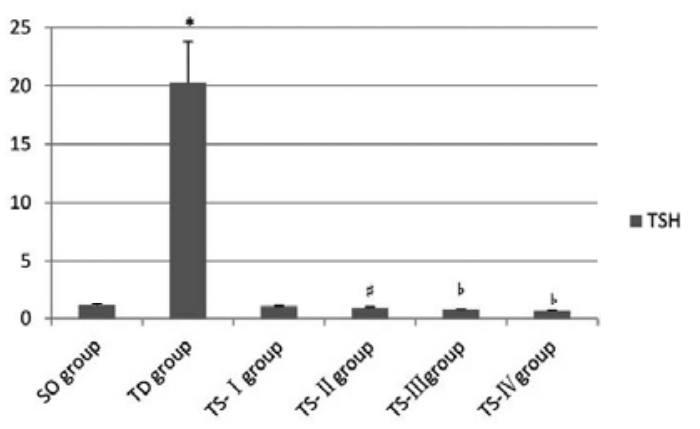

Fig. 4 Serum levels of thyroid hormone

A: Serum levels of T3 in rats in each group ( ${ }^{*} \mathrm{P}<0.01$ vs. SO group; ${ }^{b} \mathrm{P}<0.01$ vs. SO group); B: Serum levels of $\mathrm{T} 4$ in rats for each group $\left({ }^{*} \mathrm{P}<0.01\right.$ vs. SO group; $\bullet \mathrm{P}<0.05$ vs. SO group; ${ }^{\mathrm{P}}<0.01$ vs. SO group; $b \mathrm{P}<0.01$ vs. SO group); C: Serum levels of TSH in rats for each group $\left({ }^{*} \mathrm{P}<0.01\right.$ vs. SO group; ${ }^{*} \mathrm{P}<0.01$ vs. SO group; $b \mathrm{P}<0.01$ vs. SO group)

(Note: Normal T3 level: 0.73-0.98 ng/mL; normal T4 level: 4.2-8.4 ng/mL; normal TSH level: 0.76-1.29 $\mu \mathrm{IU} /$ $\mathrm{mL})$

ing thyroid tissue, as this causes bleeding and affects the clarity of the surgical field of vision, which can increase the risk of recurrent laryngeal nerve injury and worsen crushing of the residual thyroid tissue in the surgical area. HE staining was undertaken in the resected tissue of the experimental rats, and excised tissues were identified as normal thyroid gland. At the same time, HE staining of neck tissue of the thyroid gland cross-section showed no follicular structure in the histology of the thyroid gland, while tracheal cartilage, smooth muscle, connective tissue, and inflammatory cells infiltrated the resected area, indicating that thyroidectomy was successfully performed and all thyroid tissues were resected.

Race, region, nutrition, iodine intake, and measurement methods all affect thyroid hormone levels, and TSH reference values vary significantly by age, gender, time of day, and race ${ }^{13,14}$. Therefore, reference values for thyroid function must be established in Wistar rats before creating a model for TSH suppression therapy after total thy- roidectomy in rats. In a previous study, we successfully established normal local reference values for serum thyroid hormones in adult Wistar rats'. This provided a normal reference value range for this study and basic data for the study on thyroid-related diseases in this region. In rat experiments, methods of exogenous administration of L-T4 included intragastric perfusion, subcutaneous injection, intraperitoneal injection, and subcutaneous minipump implantation ${ }^{15-19}$. The advantage of oral administration is that it is easy and does not cause irritation to rats; however, it cannot properly control the dose, which is easily affected by food intake (or water intake) and the digestive function of rats. Intraperitoneal injection can accurately control drug dosing; however, rapid drug absorption leads to marked fluctuations in T3 and T4 levels in plasma and tissue. Unlike intraperitoneal injection, subcutaneous mini-pump injection of L-T4 into the backs of rats does not cause drastic fluctuation of thyroid hormone level; however, the dose cannot be adjusted in rela- 
tion to BW. Because hypothyroidism can increase BW in rats after thyroidectomy, the change in BW might lead to a mismatch between dose and BW. For this reason, we selected a method of subcutaneous injection of L-T4 that allows for accurate control of dose and adjustment at any time in relation to change in BW. Although it can still cause fluctuations in thyroid hormone levels in rats, subcutaneous drug absorption is slower than in the abdominal cavity, and fluctuations of thyroid hormone levels are more moderate in rats.

In addition to choosing an appropriate method for administration, the dose and duration of administration are important. In a previous study, L-T4 was injected at a daily dose of $1.6 \mu \mathrm{g} / 100 \mathrm{~g}$ BW, which supplemented reduced thyroxine hormone and replaced thyroid function in rats $^{20}$. Therefore, in the present study we selected 4 doses of L-T4-1.4, 1.6, 1.8, and $2.0 \mu \mathrm{g}$ per $100 \mathrm{~g}$ BW-after thyroidectomy. Van et al. confirmed that after rats were injected with radioisotope-labeled T4 for 6 to 8 days, the total amount of labeled metabolites excreted through feces and urine became stable and was equal to the daily injected dose of $\mathrm{T} 4^{21}$. However, in other studies, after total thyroidectomy, L-T4 was injected into rats for 12 to 13 days to achieve replacement ${ }^{22}$. On the basis of the above findings, in this study serum thyroid hormones were tested after 15 days of continuous injection of L-T4. The results confirmed that administration of L-T4 $1.6 \mu \mathrm{g} / 100$ g BW to rats for 15 consecutive days after thyroidectomy was optimal for an animal model for TSH suppression therapy. The administered dose of L-T4 for replacement therapy was slightly different from the results of the above studies, perhaps because of the effects of region, iodine intake, and measurement method on thyroid function. Inner Mongolia is dry and cold, so normal reference values for serum thyroid hormone in adult Wistar rats are different from those in other areas. Similarly, iodine intake was lower than in other regions. Our results show the dose of L-T4 required for TSH suppression therapy in Wistar rats after thyroidectomy.

In conclusion, rats subcutaneously injected with L-T4 at a dose of $1.6 \mu \mathrm{g} / 100 \mathrm{~g}$ BW for 15 days after total thyroidectomy were suitable for use as an animal model for TSH suppression therapy.

Conflict of Interest: The authors declare no conflicts of interest.

\section{References}

1. Siegel RL, Miller KD, Jemal A. Cancer statistics, 2020. CA
Cancer J Clin. 2020;70(1):7-30.

2. Jin S, Yang YT, Bao W. Signaling pathways in thyroid cancer. Vitam Horm. 2018;106:501-15.

3. Carhill AA, Litofsky DR, Ross DS, et al. Long-term outcomes following therapy in differentiated thyroid carcinoma:NTCTCS registry analysis 1987-2012. J Clin Endocrinol Metab. 2015;100(9):3270-9.

4. Kim HI, Jang HW, Ahn HS, et al. High serum TSH level is associated with progression of papillary thyroid microcarcinoma during active surveillance. J Clin Endocrinol Metab. 2018;103(2):446-51.

5. Jonklaas J, Sarlis NJ, Litofsky D, et al. Outcomes of patients with differentiated thyroid carcinoma following initial therapy. Thyroid. 2016;16(12):1229-42.

6. Do Cao C, Wémeau JL. Risk-benefit ratio for TSH- suppressive Levothyroxine therapy in differentiated thyroid cancer. Ann Endocrinol(Paris). 2015;76(1 Suppl 1):1S47-52.

7. Blum MR, Bauer DC, Collet TH, et al. Subclinical thyroid dysfunction and fracture risk: a meta-analysis. JAMA. 2015;313(20):2055-65.

8. Jin S, Yang YT, Bao W, et al. Naming difficulties after thyroid stimulating hormone suppression therapy in patients with differentiated thyroid carcinoma: a prospective cohort study. Endocrine. 2019;65(2):327-37.

9. Liu Y, Jin S, Ai J, et al. Reference range of serum thyroid hormones in healthy adult rats in Inner Mongolia Autonomous Region. Chin J Clinic. 2018;12:337-40.

10. Bai $Y$, Jin $S$, Ta L, et al. Establishment of a rat model of total thyroidectomy. Chin J Clinic. 2018;12:333-6.

11. Nilsson M, Fagman H. Development of the thyroid gland. Development. 2017;144(12):2123-40.

12. Sola-Marti R, Mlas-Carballal C. Anatomic description of the course of recurrent nerves in the rat. Bull AssocAnat (Nancy). 1995;79(245):29-32. 79:29-32.

13. Ehrenkranz J, Bach PR, Snow GL, et al. Circadian and circannual rhythms in thyroid hormones: Determining the $\mathrm{TSH}$ and free T4 reference intervals based upon time of day, age, and sex. Thyroid. 2015;25(8):954-61.

14. Jeon MJ, Kim WG, Kwon $\mathrm{H}$, et al. Excessive iodine intake and TSH reference interval: Data from the Korean National Health and Nutrition Examination Survey. Thyroid. 2017;27(7):967-72.

15. Akhondali Z, Badavi M, Dianat M, Faraji F. Coadministration of apelin and $\mathrm{T} 4$ protects inotropic and chronotropic changes occurring in hypothyroid rats. Arq Bras Cardiol. 2015;105:235-40.

16. Shabani S, Sarkaki A, Ali Mard S, et al. Central and peripheral administrations of levothyroxine improved memory performance and amplified brain electrical activity in the rat model of Alzheimer's disease. Neuropeptides. 2016;59:111-6.

17. Hung PL, Huang CC, Huang HM, Tu DG, Chang YC. Thyroxin treatment protects against white matter injury in the immature brain via brain-derived neurotrophic factor. Stroke. 2013;44:2275-83.

18. Zhang Y, Dedkov EI, Lee B $3^{\text {rd }}$, Li Y, Pun K, Gerdes AM. Thyroid hormone replacement therapy attenuates atrial remodeling and reduces atrial fibrillation inducibility in a rat myocardial infarction-heart failure model. J Card Fail. 2014;20:1012-9.

19. Klein I, Danzi S. Evaluation of the therapeutic efficacy of different levothyroxine preparations in the treatment of human thyroid disease. Thyroid. 2003;13:1127-32.

20. Samadi R, Ghanbari M, Shafiei B, Gheibi S, Azizi F, Ghasemi A. High dose of radioactive iodine per se has no 
effect on glucose metabolism in thyroidectomized rats. Endocrine. 2017;56:399-407.

21. Van DJ, Van d HD, Roelfsema F. Sources and quantity of 3,5,3'-triiodothyronine in several tissues of the rat. J Clin Invest. 1983;72:1778-92.

22. Escobar-Morreale HF, Obregón MJ, Escobar F, Morreale G. Replacement therapy for hypothyroidism with thyroxine alone does not ensure euthyroidism in all tissues, as studied in thyroidectomized rats. J Clin Invest. 1995;96: 2828-38.
(Received, May 13, 2020)

(Accepted, July 31, 2020)

(J-STAGE Advance Publication, August 31, 2020)

Journal of Nippon Medical School has adopted the Creative Commons Attribution-NonCommercial-NoDerivatives 4.0 International License (https://creativecommons.org/licenses/by-nc-nd/4.0/) for this article. The Medical Association of Nippon Medical School remains the copyright holder of all articles. Anyone may download, reuse, copy, reprint, or distribute articles for non-profit purposes under this license, on condition that the authors of the articles are properly credited. 